\title{
Potential of Interplanetary Torques and Solar Modulation for Triggering Terrestrial Atmospheric and Lithospheric Events
}

\author{
Rhodes W. Fairbridge \\ Columbia University \& NASA/Goddard Institute for Space \\ Studies, New York 10025, USA \\ Göran Windelius \\ Motjärnshytten 44, S-68391 Hagfors, Sweden \\ Hans J. Haubold \\ UN Outer Space Office, Vienna International Centre, \\ P.O.Box 500, A-1040 Vienna, Austria
}

\begin{abstract}
The Sun is forced into an orbit around the barycenter of the solar system because of the changing mass distributions of the planets. Solarplanetary-lunar dynamic relationships may form a new basis for understanding and predicting cyclic solar forcing functions on the Earth's climate.
\end{abstract}


Using the NASA-Jet Propulsion Laboratory planetary ephemeris DE-102, a time-tabel of paired synodic conjunctions has been prepared for the four major planets extending more than $16.000 \mathrm{yr}$ in the past and $100 \mathrm{yr}$ into the future (Fairbridge and Sanders, 1987; Sanders and Fairbridge, 1987). While all planetary orbital periods are somewhat irregular over short-term intervals, when calculated over time periods $>1000 \mathrm{yr}$, long-term means establish a pattern of beat frequencies that conform to a complex harmonic system with an approximately $\log / \log$ relationship with a constant slope. The slope formula, where $\mathrm{v}$ denotes harmonic and $\mathrm{u}$ denotes the period (in years), is as follows:

$$
\log (v)=6.04-\log (u)
$$

Without exception, all beat frequencies and resonance periods are commensurable (in simple, whole-integer ratios), subject to "errors" that decrease systematically over greater time intervals $\left(10^{-5}\right.$ at $\left.>10^{4} \mathrm{yr}\right)$. For the lunar planets, comparable pair beat frequencies are found in the spectral analysis of monthly sunspot numbers (Currie, 1973) and in the time domain the numbers have been found to correspond to the ephemeris predictions (Verma, 1986). 
This astronomical time-table provides a basis for comparison with the history of behavior patterns of (Fairbridge, 1989; Shirley, Sperber, and Fairbridge, 1990; Haubold and Beer, 1992):

(a) The Sun: monitored over $>90.000$ yr by use of ${ }^{18} \mathrm{O}$ isotopes in ice cores and deep-sea deposits; over nearly 10.000 yr by use of ${ }^{14} \mathrm{C}$ and ${ }^{10} \mathrm{Be}$ isotopes in tree rings and ice cores; over $>2000 \mathrm{yr}$ by observations of auroras and sporadic sunspot events, over $>300$ yr by telescopic sunspot data; and during the 20th century by multidisciplinary sensing systems, most recently from satellite platforms.

(b) The Earth: monitored over $>4$ billion years through the geological record (which, through evidence of mineral equilibria, sedimentological mechanisms and the uninterrupted continuum of organic evolution, conclusively proves the fundamental stability of the Solar System); monitored over the 560 million year Phanerozoic interval by analysis of sequence stratigraphy (suggesting the stability of the Earth/Moon orbital relationships in the 20, 41, 93 kyr "Milankovitch" cyclicity).

Calibration of planetary/Sun/Earth relationships has been obtained using the chronometry of the integrated tree ring record (accurate to $\pm 1 \mathrm{yr}$ ), and supplemented by ice core data, both of which furnish isotopic data reflecting 
(1) Sun's emissions, (2) Earth's spin rate, axis parameters, and geomagnetic variables. Both, in turn apply forcing to the Earth's climatic dynamics.

Planetary triggering of dynamic events on the Sun and Earth is believed to operate primarily through exchange of interplanetary torques. Although the Saturn-Jupiter pair (synodic lap: 19.859 yr) carry $86 \%$ of the angular momentum of the Solar System, it is the synod of the two outermost giants (Neptune-Uranus: lap $171.4095 \mathrm{yr}$ ) that acts as the primary trigger to a sporadic ripple effect that propagates through the system. Other pairs (USL, SJL, EVL, etc.) set up secondary ripples. The closest conjunctions are extremely rare, but create a serial domino effect through the system.

On planet Earth two " control dates" have been selected that help provide a framework for constructing a climatic chronology. For warm cycles the best data is -1090 (sidereal) or 3040 B.P. (before A.D. 1950), principal fluctuations occur at multiples of 171.4 yr (NUL $\times 3,9,27$, etc.). For the cold cycles the date is -3321 or 5271 B.P., together with the principal multiples (at NUL $\times$ $2,6,12$, etc.).

It is now postulated that this knowledge of long-term historical behavior constitutes a powerful tool for estimating future hazardous events on planet Earth, e.g. seismic crescendos, volcanic eruptions, climatic crises. The year 
AD 1993 was long recognized a an NUL synod and closely clustered ( $\pm 5 \mathrm{yr}$ ) with other pairs. The $171.4 \mathrm{yr}$ cycle is $\pm 1.4 \mathrm{yr}$; 1993 is $18 \times \mathrm{NUL},+2 \mathrm{yr}$, after -1090. It did in fact turn out to be an "interesting" year. A much more refined assessment of astronomical triggering of terrestrial hazard potentials should now be possible. 


\section{Acknowledgements}

The NASA-Goddard Institute for Space Studies (New York City) has materially aided this research in many ways, also assisted by an NSF (Engineering)

grant (R.W.F., 1988/90).

\section{References}

Currie, R.G.: 1973, Astrophys. Space Sci. 20, 509.

Fairbridge, R.W.: 1989, Quaternary International 2, 83.

Fairbridge, R.W. and Sanders, J.E.: 1987, in M.R. Rampino, J.E. Sanders, W.S. Newman, and L.K. Königsson (eds.), 'Climate: History, Periodicity, Predictability', Van Nostrand Reinhold, New York, pp. 446-471,

Haubold, H.J. and Beer, J.: 1992, in W. Schröder and J.P. Legrand (eds.), 'Solar-Terrestrial Variability and Global Change', Selected papers from the symposium of the Interdivisional Commission on History (ICH) of the International Association of Geomagnetism and Aeronomy (IAGA), XX General Assembly of IUGG, Vienna, Austria, 11-24 August 1991, pp. 11-34. 
Sanders, J.E. and Fairbridge, R.W.: 1987, in M.R. Rampino, J.E. Sanders, W.S. Newman, and L.K. Königsson (eds.), 'Climate: History, Periodicity, Predictability', Van Nostrand

Reinhold, New York, pp. 475-541.

Shirley, J.H., Sperber, K.R., and Fairbridge, R.W.: 1990, Solar Phys. $\underline{127}, 379$. 